\title{
Estimation of kidney tumor volume in CT images using medical image segmentation techniques
}

\author{
Faleh H. Mahmood ${ }^{1}$, Nada A. Mahmood ${ }^{2}$, Abdul Rahman H. Ismaeel ${ }^{3}$
}

${ }^{1}$ Remote Sensing Unit, College of Science, University of Baghdad, Baghdad, Iraq

${ }^{2}$ Abi Greabe Hospital, Baghdad Health Directorate - Al-Karkh, Ministry of Health,

Baghdad, Iraq

${ }^{3}$ Department of Physics, College of Science, University of Baghdad, Baghdad, Iraq

E-mail: faleh_sine@yahoo.com

\begin{abstract}
Kidney tumors are of different types having different characteristics and also remain challenging in the field of biomedicine. It becomes very important to detect the tumor and classify it at the early stage so that appropriate treatment can be planned. Accurate estimation of kidney tumor volume is essential for clinical diagnoses and therapeutic decisions related to renal diseases. The main objective of this research is to use the Computer-Aided Diagnosis (CAD) algorithms to help the early detection of kidney tumors that addresses the challenges of accurate kidney tumor volume estimation caused by extensive variations in kidney shape, size and orientation across subjects.

In this paper, have tried to implement an automated segmentation method of gray level CT images. The segmentation process is performed by using the Fuzzy C-Means (FCM) clustering method to detect and segment kidney CT images for the kidney region. The propose method is started with pre-processing of the kidney CT image to separate the kidney from the abdomen CT and to enhance its contrast and removing the undesired noise in order to make the image suitable for further processing. The resulted segmented CT images, then used to extract the tumor region from kidney image defining the tumor volume (size) is not an easy task, because the 2D tumor shape in the CT slices are not regular. To overcome the problem of calculating the area of the convex shape of the hull of the tumor in each slice, we have used the Frustum model for the fragmented data.
\end{abstract} Key words

Image segmentation, CT image, Fuzzy CMeans (FCM), kidney tumor volume.

$$
\begin{aligned}
& \text { تقدير حجم الورم الكلوى في الصور المقطعية باستخدام تقنيات تقبيم الصور الطبية } \\
& \text { فالح حسن محمود1، ندى عبد الحافظ محمود2، عبدالرحمن حميد اسماعيل } 3
\end{aligned}
$$

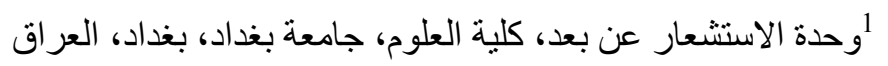

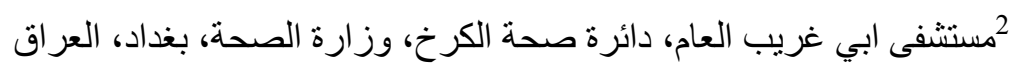

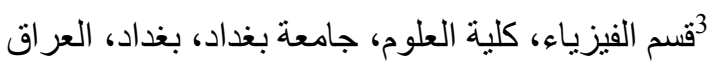

Article info.

Received: Dec. 2017

Accepted: Jan. 2018

Published: Jun. 2018 


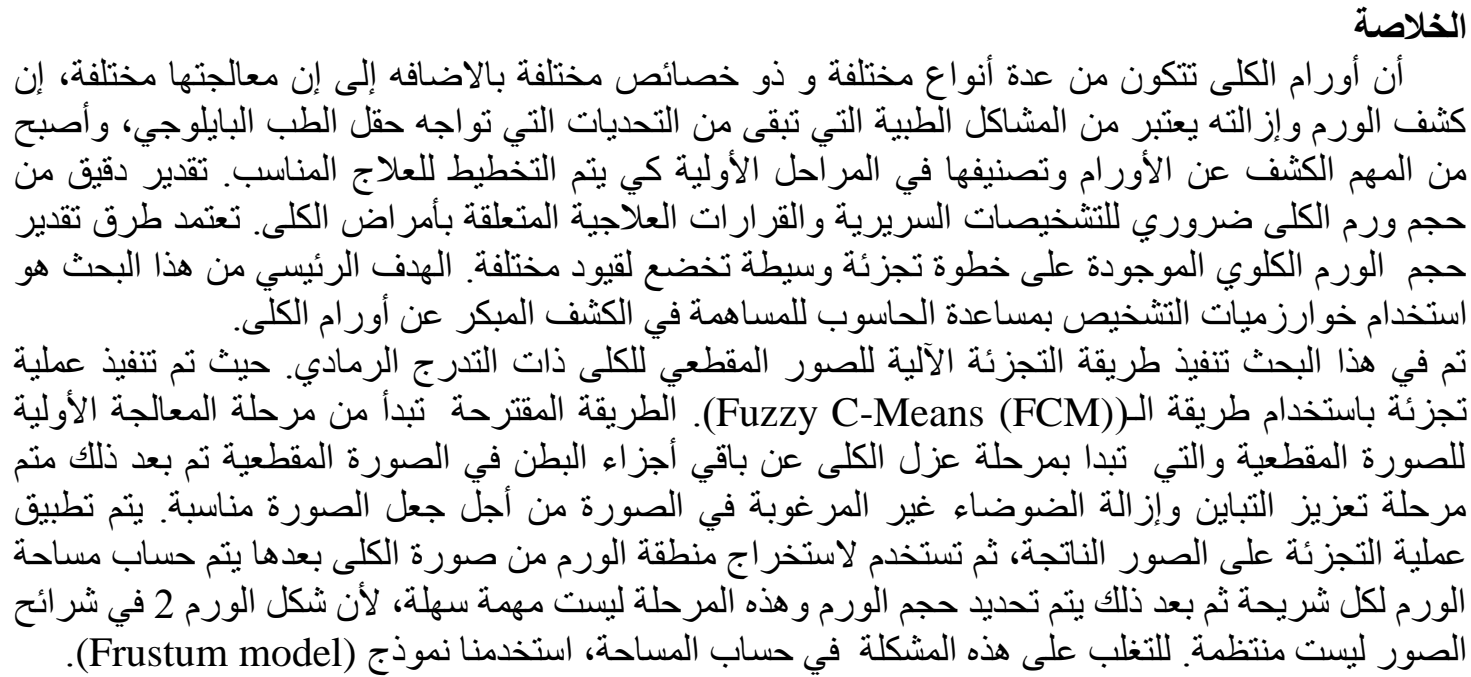

\section{Introduction}

Kidneys cancer is one of the most common cancers worldwide, with increasing morbidity and high mortality [1]. Computed Tomography (CT) has been widely used for clinical diagnosis of hepatic disease because of its high resolution. Accurate kidneys segmentation from abdominal CT scans is critical for computer-assisted diagnosis CAD and therapy, including patient specific kidneys anatomy evaluation, functional assessment, treatment planning, and image-guided surgery [2].

Traditionally, radiologists or physicians have to manually delineate the kidneys region slice by slice, which is tedious and time-consuming due to the large amount of data. Therefore, accurate and efficient methods for kidneys segmentation are demanded. Image segmentation is the process of partitioning a digital image into multiple segments or set of pixels. The objective of image segmentation is to group pixels into a prominent image region. Medical Image segmentation is one of most important issues in medical technology, which assists physicians in various aspects, such as analysis and diagnosis of different diseases, the study of anatomical structure, making treatment planning. It refers to the process of deriving meaningful regions from medical images that are homogeneous with respect to local image features such as edges, texture, and color, etc. With the increase of CT images in the diagnosis and treatment of diseases, segmentation of human organs from CT images is a prerequisite step in the precise treatment planning. Kidney segmentation from CT images remains an open challenge due to the high variability in the shape and size of kidney, presence of pathologies like tumor or cirrhosis, and low contrast with adjacent tissues or organs. Recently, a large variety of semiautomatic and automatic methods have been developed to improve the kidney segmentation procedure [3].

Among these useful applications are; re-constructing 3D images from set of 2D segments, improving image contrast and removing noise and blurriness, performing quantitative analysis useful for pathogens, as well as a superior ability for early diagnosis. The most frequently steps used in the medical image processing methods are image registration, which aims at aligning image data from different modalities, subjects, or points of time, as well as image segmentation which localize and delineate the relevant objects in 2D projections and transform them into 3D visualization 
[4]. Computerized axial tomography (CT) provides a 2D map of the line attenuation coefficient mirroring morphological details of the organs under study. It has high spatial resolution and wide dynamic range of imaging modalities, so, even small density abnormalities can be detected. A CT image can be obtained within one breath hold which makes CT the modality of choice for imaging the thoracic cage [5].

However, image segmentation is an important tool in medical image processing, and has a very useful impact in many applications. The applications include detection of the coronary border in angiograms, multiple sclerosis lesion quantification, surgery simulations, surgical planning, measurement of tumor volume and its response to therapy, etc. The segmentation methods are, frequently, divided into different classes, depending on classification scheme: Manually (semiautomatic and automatic), Pixel-based and regionbased methods, Manual delineation (e.g. thresholding, region growing), Classical (edge-based and region-based techniques) e.g. statistical, fuzzy, and neural network techniques. The most commonly used segmentation techniques thus are classified into two broad categories Region segmentation techniques that look for the regions satisfying a given homogeneity criterion, and edge-based segmentation techniques that look for boundary regions between different characteristics areas [6].

In our proposed work, have be using image segmentation technique based on the fuzzy C-Means algorithm (FCM) for kidney segmentation to segment the kidney region. The Fuzzy C-Means (FCM) clustering algorithm generalizes the hard c-mans algorithm to allow a point to partially belong to multiple clusters. Therefore, it produces a soft partition for a given dataset. In fact, it produces a constrained soft partition. Watershed transform is a powerful tool for image segmentation; it is a region based approach of segmentation [7]. The image slices can then be stacked on top of one another, creating a 3D image volume. Using physical spacing information inherent in the image slices, it is possible to reconstruct the tumor from the segmented data as well as estimate the tumor volume [6].

\section{The samples of kidney images}

The medical data used for algorithm evaluation consists of $2 \mathrm{D}$ abdominal CT scan data from different healthy and unhealthy patients of mixed gender (Men, Women) and age (25 to 55 years old). Different samples of abdomen CT scan images are collected from the local hospitals (Baghdad Teaching Hospital and, General Abi-Graieb Hospital) / Ministry of health (some samples of normal and abnormal for CT images). The data was acquired using SIEMENS CT scanners sensation 16 with a resolution in $\mathrm{x} / \mathrm{y} / \mathrm{z}$ from $0.6 / 0.6 / 5$ to $0.75 / 0.75 / 5$ (in $\mathrm{mm}$ ) and provided in DICOM format. The parameters of CT scan images for scanning were $120.0 \mathrm{KV}$ and 297.0 $\mathrm{mA}$. The pixel spacing was 0.683594 $\mathrm{mm}$, the slice thickness was $5.0 \mathrm{~mm}$ and the spacing between slices was 0.3 $\mathrm{mm}$. The number of slices ranged from 65 to 140 . CT images of the kidney are processed for the detection of tumor using MATLAB. In this project, only one patients' abnormal samples CT scan slice images each slice of these datasets had a spatial resolution of $256 \times 256$ pixels was selected from different samples mentioned .The samples images have bitmap BMP format with color depth $24 \mathrm{bit} / \mathrm{pixel}$; and the size of each image is $256 \times 256$ pixels, each of thickness ranged $5 \mathrm{~mm}$. The segmentation 
experiments and performance evaluation were carried of kidney CT images were run on the 64-bit on the computer with Pentium Intel (R)-Core (TM) i5, CPU (2.5 GHz) and $3 \mathrm{~GB}$ memory.

\section{Image analysis system}

In our proposed work for automated method to segment kidney and detect tumor, the estimation of kidney tumor volume for CT images was introduced, as the block diagram is shown in Fig. 1 illustrated the steps of imaging analysis system adopted of the basic processes which is include; after image acquisition, the pre-processing is first stage the next stage is image segmentation, in third stage tumor region extraction, finally accurate estimation of kidney tumor volume is the last stage descript in bellow.

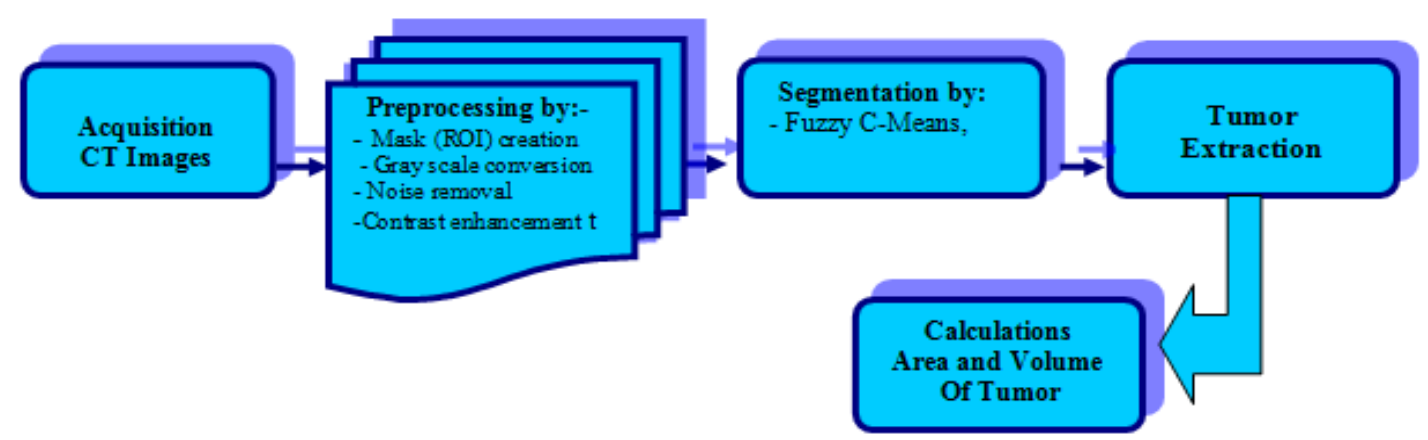

Fig.1: The block diagram of the basic processes.

\section{Pre-processing stage}

In this part of the work, firstly separate the kidney region from the abdomen CT Images. Therefore, the first stage in any recognition system is pre-processing. It is the name for operations on images at the lowest level of abstraction whose aim is an improvement of the image data that remove undesired distortions or enhances some image features important for further processing. So, the goal of pre-processing is to remove the noise and to provide contrast enhancement to improve the image quality. The functions performed by pre-processing process are. Mask Region of Interest (ROI) creation, Gray scale conversion, Noise removal and contrast enhancement.

First, the abdominal CT image is pre-processed using the median filter. Then contour points were selected manually from the abdominal CT images for isolate kidney portions.
The original abdominal CT images are considered for masking to find the variation in pixel intensities between the region of interest and the background of the image. Kidney regions are extracted after executing the masking module. Fig. 2 shows a sample of the mask. So in order separates the kidney from the abdomen CT Image it's important to create a mask which is a binary image $(0$ or 1 's). This mask is used to subtract the unwanted features in the images. The mask which has taken the size of the kidney, its value and shape differ from one image to another. It is applied on the original abdomen $\mathrm{CT}$ image to discard the irrelevant information multiplying the mask image with study image it produced the masked image which is the kidney image. The kidney appears white which the value of (1) is and the background which is the reaming information appears (0). 


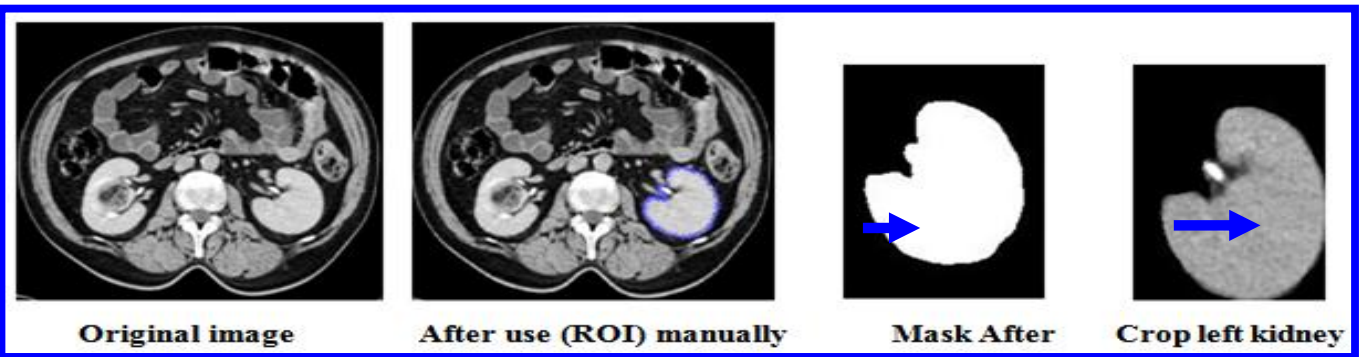

Fig. 2: The mask creation performed to crop the left kidney region.

Since the CT images consist of primary colors (RGB) content therefore the next step in preprocessing stage which is the grayscale conversion. A gray color is one in which the red, green and blue components all have equal intensity in RGB space and so it is only necessary to specify a single intensity value for each pixel, as opposed to the three intensity values needed to be specified for each pixel in a full color image. When CT images are viewed, they look like black and white but they contain some primary colors (RGB) [8]. So, further processing of CT kidney image, it must be converted to perfect grayscale image, as shown in Fig. 3.

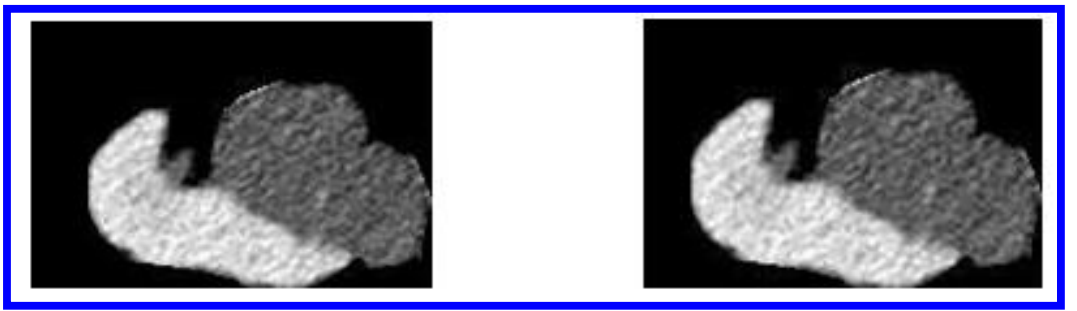

Fig. 3: Conversion from RGB to grayscale image.

The common noises present in a renal, ureter, and bladder CT scan are salt \& pepper noise, speckles, Gaussian and impulse noises [9]. In CT image, salt and pepper noise and Gaussian are more predominant. We apply nonlinear median filter to remove the salt and pepper noise, whereas Gaussian noise is eliminated by a Gaussian high pass filter. The median filter is used to reduce the salt and pepper noise present due to motion artifacts (i.e.
Movement of patient during scan) in the CT images. It is done for smoothening of CT image. Here we are using CT median filters to eliminate salt and pepper noise. A high pass filter preserves the high frequency information within an image while reducing the low frequency information, thus emphasizing the transitions in the image intensities, as shown in Fig. 4.

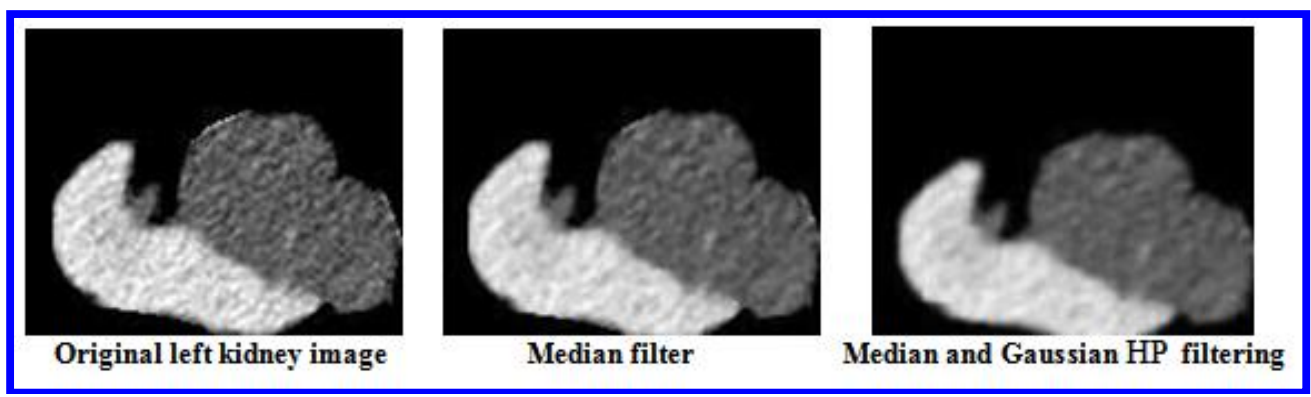

Fig. 4: Pre-processing technique after applied median and Gaussian HP filter. 


\section{Fuzzy C-Means (FCM) image segmentation}

In this section, the proposed kidney segmentation methods with a hierarchical strategy will be presented in detail. The Fuzzy C-Means (FC) method was implemented to detect and extract tumors and abnormalities in CT scan kidney images.

The fuzzy logic is used to process data by giving the partial membership value to each pixel in the image. The membership value of the fuzzy set ranges between 0 and 1 . Clustering method is a kind of unsupervised learning. So the segmentation methods based on it do not need training sample data, they form clusters of data by grouping pixels. Fuzzy clustering is a multi-valued logic system that uses intermediate values i.e., member of one fuzzy set can also be member of another fuzzy set while in the same image. There are no discontinuous or sudden transitions between full membership and non-membership functions. The membership function defines the fuzziness of an image and the information contained in the image. The primary features involved in characterization using a membership function are: core, support, and boundary. The core is completely a member of the fuzzy set. The support is non membership value of the set and boundary is the partial membership value, having its value between 0 and 1. This algorithm works by assigning membership to each data point corresponding to each cluster center on the basis of distance between the cluster center and the data point. More the data is near to the cluster center, more is its membership towards the particular cluster center. Hence, addition of membership of each and every data point must be equal to one [7].
The idea of fuzzy connectedness goes back to the work of Rosenfeld. Thus improvement in segmentation and tumor detection can be done with Fuzzy C-Means clustering method. Fuzzy C-Means (FCM) clustering is an unsupervised technique that has been successfully applied to feature analysis, clustering, and classifier designs in fields such as astronomy, geology, medical imaging, target recognition, and image segmentation. An image can be represented in various feature spaces, and the FCM algorithm classifies the image by grouping similar data points in the feature space into clusters. This clustering is achieved by iteratively minimizing a cost function that is dependent on the distance of the pixels to the cluster centers in the feature domain. The pixels on an image are highly correlated, i.e. the pixels in the immediate neighborhood possess nearly the same feature data. Therefore, the spatial relationship of neighboring pixels is an important characteristic that can be of great aid in imaging segmentation. General boundary detection techniques have taken advantage of this spatial information for image segmentation. However, the conventional FCM algorithm does not fully utilize this spatial information. So improvement in conventional method can be done by incorporating noise reduction method also. The algorithm is an iterative optimization that minimizes the cost function defined as follows [10]:

\section{Mathematical representation}

Algorithmic steps for Fuzzy c-means clustering:

Let $X=\left\{x_{1}, x_{2}, x_{3} \ldots, x_{n}\right\}$ be the set of data points and $\mathrm{V}=\left\{\mathrm{v}_{1}, \mathrm{v}_{2}, \mathrm{v}_{3} \ldots, \mathrm{v}_{\mathrm{c}}\right\}$ be the set of centers. 
1) Randomly select 'c ' cluster centers.

2) calculate the fuzzy membership ' $\mu_{i j}{ }^{\prime}$ using:

$$
\mu_{i j}=1 / \sum_{k=1}^{c}\left(d_{i j} / d_{i k}\right)^{(2 / m-1)}
$$

$3)$ compute the fuzzy centers ' $v_{j}^{\prime}$ using:

$$
v_{j}=\left(\sum_{i=1}^{n}\left(\mu_{i j}\right)^{m} x_{i}\right) /\left(\sum_{i=1}^{n}\left(\mu_{i}\right)^{m}\right), \forall j=1,2, \ldots . c
$$

4) Repeat step 2) and 3) until the minimum ' $J$ ' value is achieved or

$\|\mathrm{U}(\mathrm{k}+1)-\mathrm{U}(\mathrm{k})\|<\beta$.

where,

$\mathrm{k}$ is the iteration step.

$\beta$ is the termination criterion between $[0,1]$.

$\mathrm{U}=\left(\mu_{\mathrm{ij}}\right) \mathrm{n}^{*} \mathrm{c}$ is the fuzzy membership matrix.

$\mathrm{J}$ is the objective function.

Implementation of the FCM segmentation can be done using the following algorithm:

Step 1: Read the kidney CT image after kidney region extracted.

Step 2: Input the number of clusters, fuzziness factor and number of iteration (default 100).

Step 3: Randomly select the initial centroid of clusters.

Step 4: Calculate the Euclidean distance between each pixel and centroid by: $d=\|x i-\theta j\|$; then find the membership function according to eq. (2).

Step 5: Find an object function according to Eq.(1).

Step 6: Compare between calculated object function according to above equation for two iterations. Stop if there is no change in membership function or cluster centers at two successive iterations. Otherwise go to Step 4: to update the membership values and cluster centroid.

Step 7: FCM method groups elements of the clusters to suitable cluster so that the distance between the element and its corresponding cluster center is minimum.
Step 8: Display the output image by find the index matrix for the maximum probability of each pixel have the same position in all clusters.

\section{Tumor region extraction}

After the segmentation is performed on kidney region, extracted that can be used as diagnostic indicators. The kidney and tumor regions are separately segmented from the CT images. The operation of tumor extraction from the result classes of Fuzzy C-Means methods can be summarized by the following steps:

Step 1: Apply Fuzzy C-means clustering method on kidney CT images in which the tumor appears.

Step 2: Binaries Fuzzy C-Means class image that contain tumor with threshold 0.7 or 0.9 (depend on the image).

Step 3: Open image to remove small objects that have fewer than $\mathrm{P}$ pixels $(\mathrm{P}$ depend on the tumor and object size), producing another binary image. The default connectivity is 8 for two dimensions.

Step 4: Repeat steps 2-3 for all samples,

Step 5: Extract only the object that has the area of tumor (depend on the sample) and eliminate other objects.

\section{Area calculation}

The area of the extracted tumor region can be computed by counting the number of pixels enclosed by the tumor region and multiplying them by the size of the pixels. The pixel's size (i.e. area) can be determined from [11]:

Pixel Size $($ Area $)=\left(\frac{\text { DFOV }}{\text { Matrix Size }}\right)^{2}$

where: DFOV is the Display Field of View (refer to how much of the scan field of view is reconstructed into an image), and matrix size represents the $2 \mathrm{D}$ grids of pixels used to compose the 
scanned images on display monitor The matrix determines the number of rows and columns.

The area of the extracted tumor region is computed by counting the number of pixels which have the value (1) in the image array. The area (A) in the object is just the count of the one's in the image array. For computing area, binary image is used [11]:

$A=n[1]$

where $\mathrm{n}$ [ ] represents the count of number of the patterns within the parenthesis

\section{Volume calculations}

The extracted tumor volume can be calculated using frustum model illustrated in Fig. 5 using [12]:

$V=\sum\left(h / 3 \times\left(A_{1}+A_{2}+\left(\frac{A_{1} \times A_{2}}{2}\right)\right)\right.$

where $\mathrm{h}($ Height $)=$ slice thickness + slice separation, The Volume V of the tumor is given by the summation of volumes of two consecutive slices. Where $A_{1}$ and $A_{2}$ denote the areas of the two consecutive slices having tumor. Calculate each slice using frustum model [12].
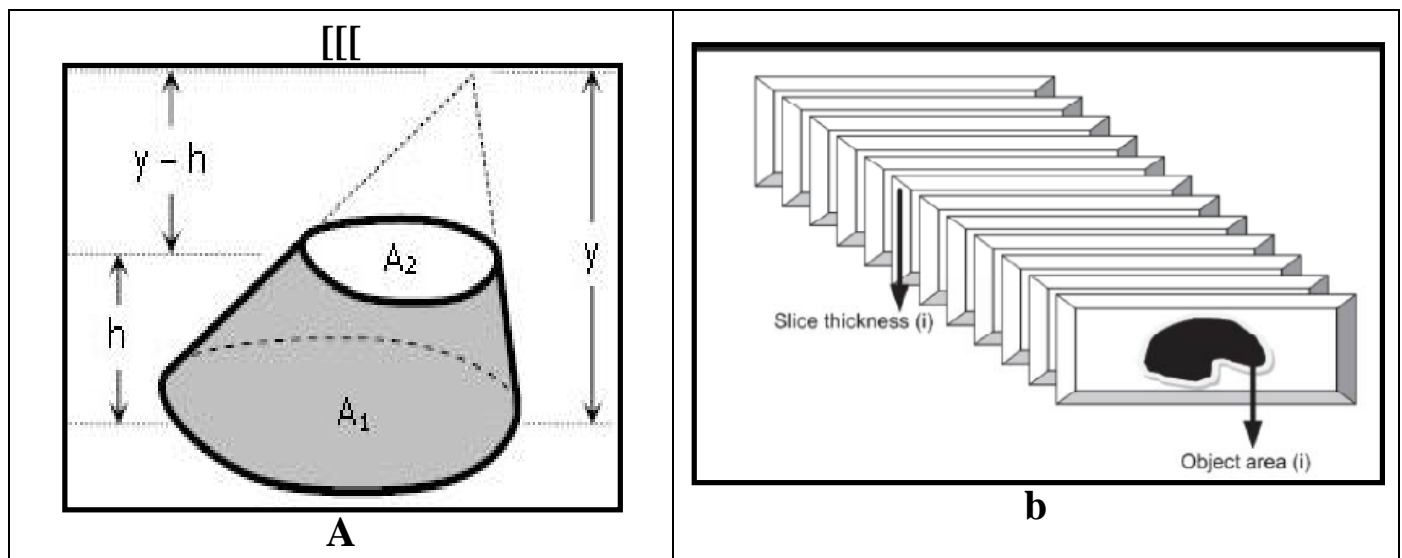

Fig. 5: Show (a) the frustum model, (b) the CT Slices [12].

The results and discussion

\section{Image segmentation and tumor extraction}

The results of kidney segmentation, in patient's CT slice images are presented and discussed in this section. In our proposed work in this research was implemented and performed only one malignant case. The number of slices in selected case ranged 56 slices. The abnormalities in the selected case appear from slice number 4 to slice number 47.

In the segmentation stage, FCM segmentation algorithm is used to detect and segment kidney CT images. Fig. 6 shows result after applied FCM clustering algorithm for one sample with 5 classes, the tumor appears in class 5 . The kidney tumor regions are separately segmented from the image as shown in Fig. 7. 


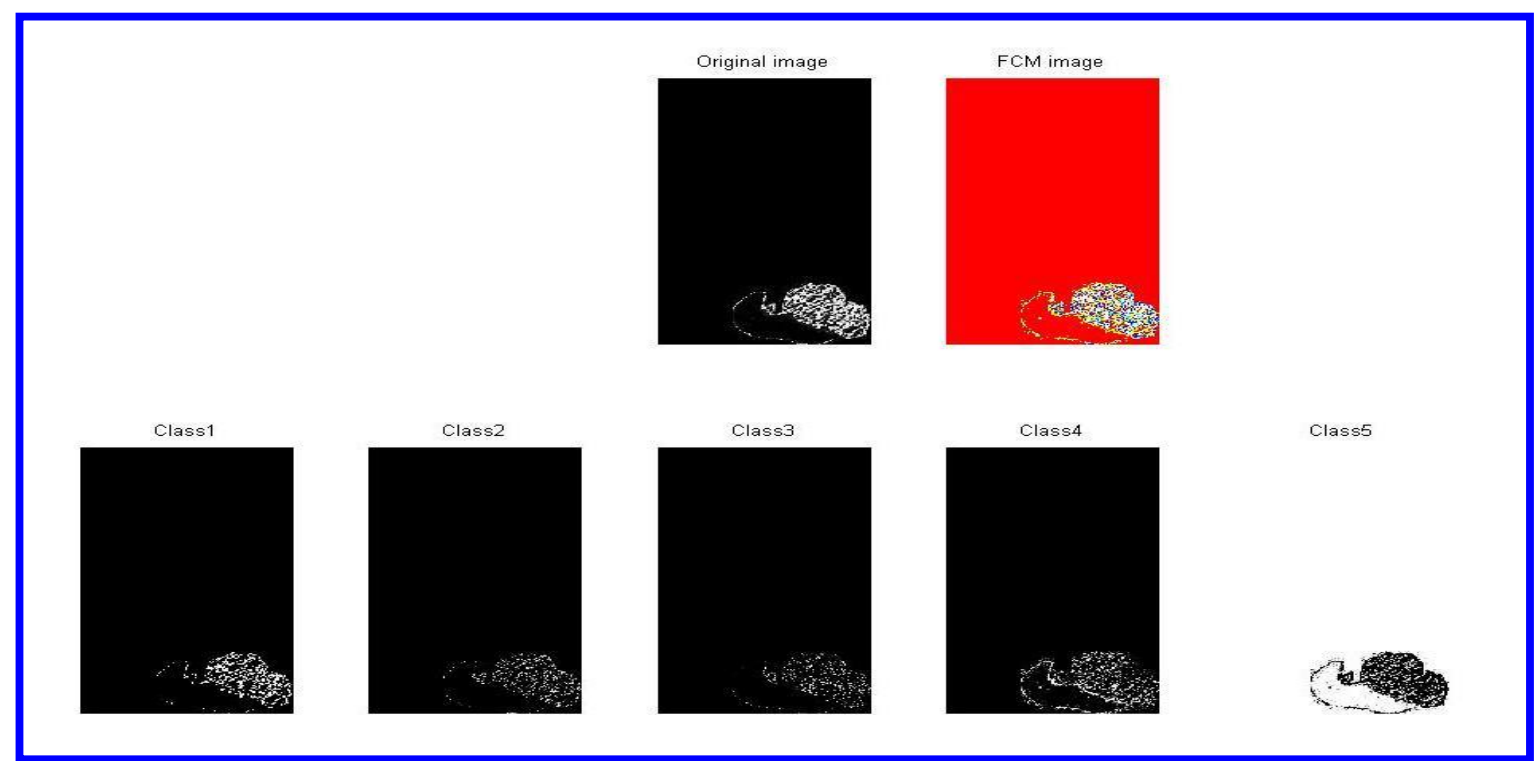

Fig. 6: FCM clustering for case1 benign (noncancerous) with 5 classes, the tumor appears in class 5.

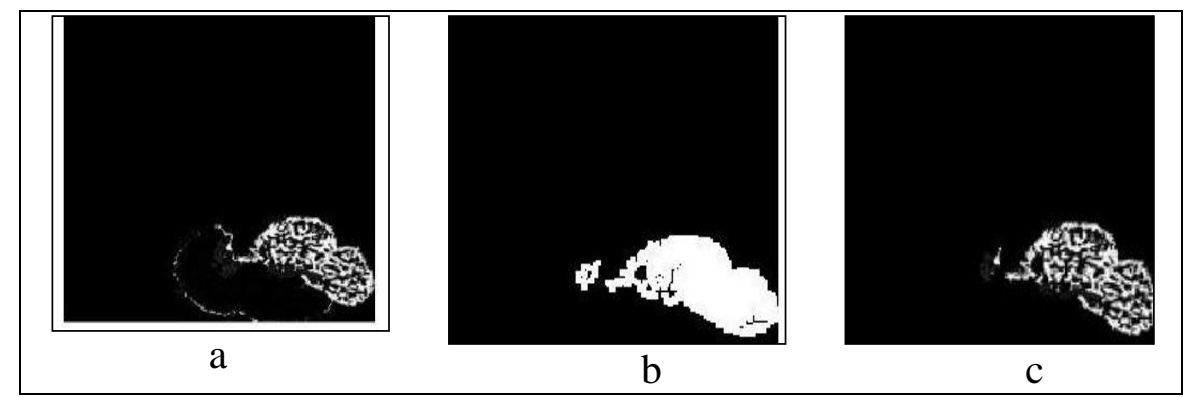

Fig. 7: a) Class of FCM include tumor, b) Binary image of extracted tumor, c) Extracted tumor.

\section{Areas and volume calculation}

A set of sequence image from case1: slice number 4 to slice 43 , with tumor region extraction based on FCM clustering segmentation method was used to calculate the area of tumor for each slice. Area of the extracted tumor is computed by counting the number of pixels which have the value (1) in the binary image. The area in pixel is converted to actual size area in $\mathrm{mm}^{2}$ by using Eq.(3). Where matrix size of CT scan $=256 \times 256$ and the field of view (FOV) of CT scan $=392 \mathrm{~mm}$. FOV and matrix size get from DICOM information of patients. Then volume of tumor calculate using frustum model between two consecutive slices with area $A i$, Eq. (4). In bellow the value of area and volume using fuzzy c-mean segmentation method this case was illustrated in Fig.8.

Table 1 illustrates the 3D tumor reconstruction processes for the patient's cases. In this table the reconstruction process slices number 4 to 47 , adopted for the reconstruction processes. The tumor extracted areas (represented by pixel's umber and in $\mathrm{mm}^{2}$ ) using Eq.(4). The matrix size of CT images was $512 \times 512$ elements, and the DFOVs were, respectively, $361 \mathrm{~mm}$ and $338 \mathrm{~mm}$ selected case. Finally, the reconstructed volume of the tumor from the extracted consecutive slice areas, using frustum model Eq.(6). 


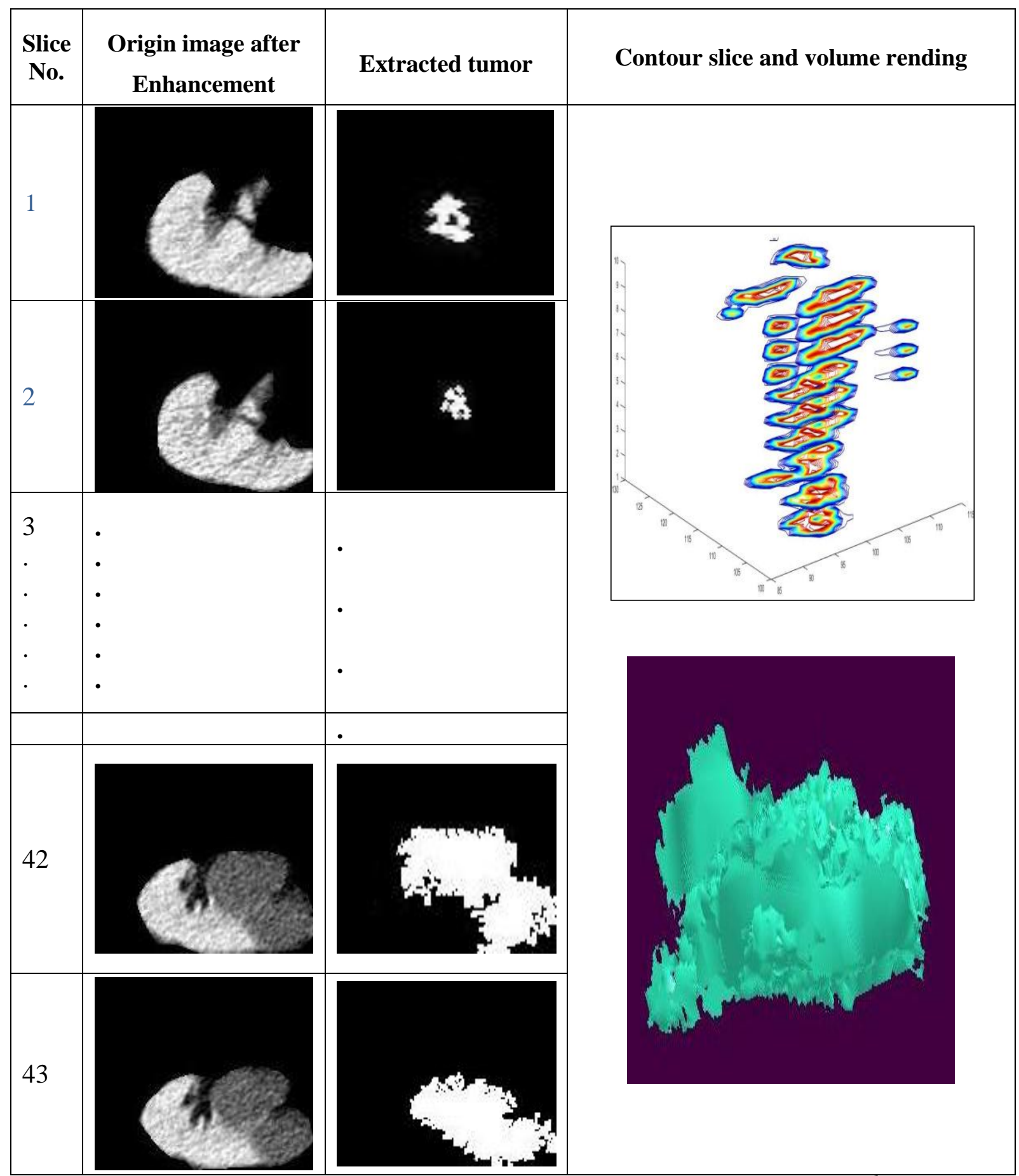

Fig.8: Contour slice and volume rending, $1^{\text {st }}$ column slice number, $2^{\text {nd }}$ column pre-processed image, $3^{\text {rd }}$ column tumor region extracted from pre-processed image, $4^{\text {th }}$ column contour slice and volume rendering of tumor region. 
Table 1: The area and volume tumor using Fuzzy $C$ - mean segmentation.

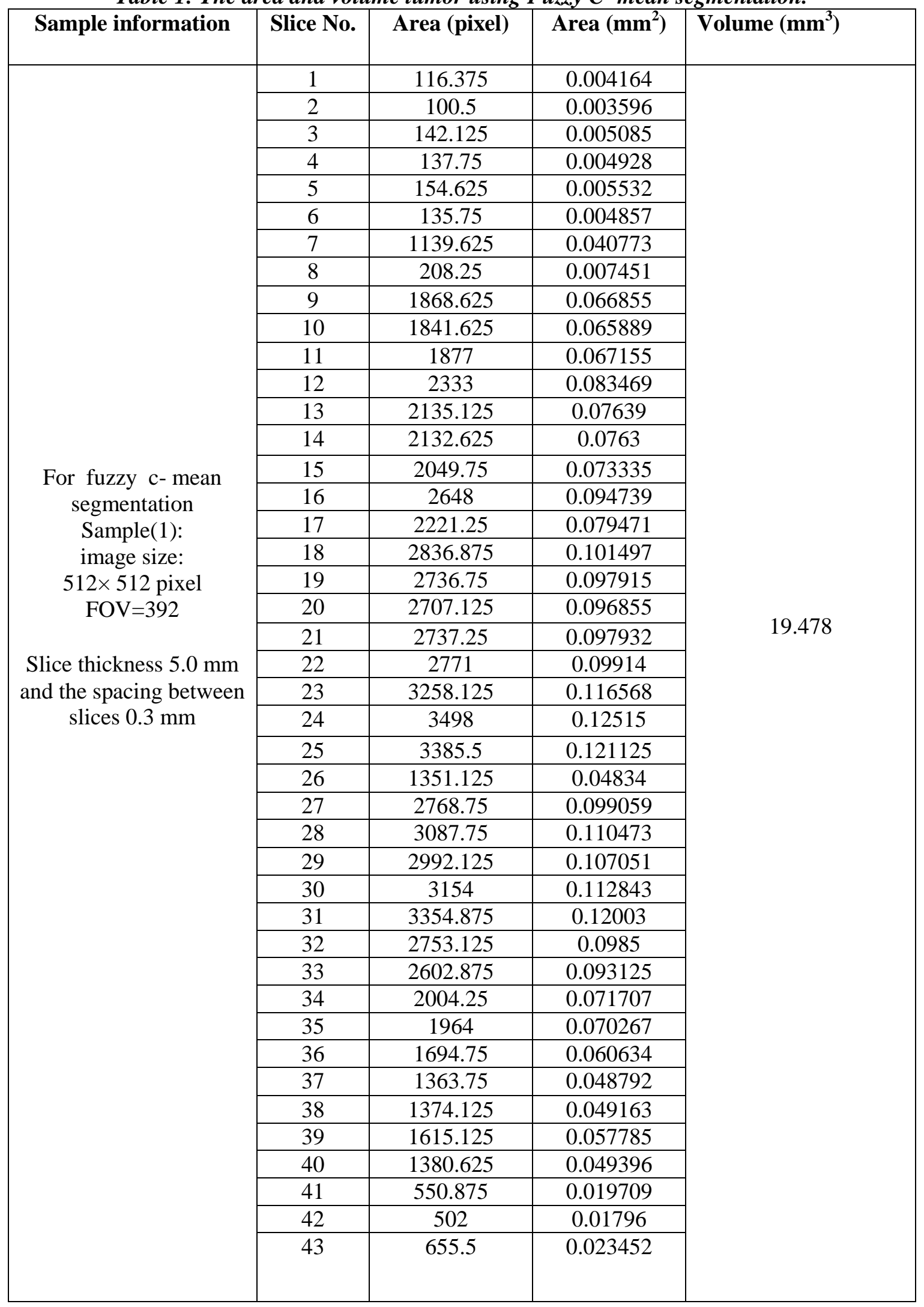

\section{Conclusions}

In this paper, an automatic segmentation method using fuzzy
C-Means for kidney tumor detection is presented. Firstly the original abdomen CT image have been pre-processed by 
segment out the kidney portion from the abdominal CT image, then enhancing their contrast to make them ready for segmentation by implementing The median filter to reduce the salt and pepper noise present due to motion artifacts (i.e. Movement of patient during scan) in the CT images to remove the salt and pepper noise present due to motion artifacts (i.e. Movement of patient during scan) in the CT images, whereas Gaussian noise is eliminated by a Gaussian high pass filter. This study shows that automated segmentation method will reduce errors occurring while doing manual segmentation. Experimental results were obtained by using MATLAB. The extracted tumor areas from kidney CT slices are measured by a method based on the Display Field of View (DFOV). The tumor areas of the image slices have been calculated and used to determine the tumor volume by stacking the extracted tumors on top of one another.

\section{References}

[1] J. Ferlay, I. Soerjomataram, R. Dikshit, S. Eser, C. Mathers, M. Rebelo, DM. Parkin, D. Forman, F. Bray, International Journal of Cancer, 136, 5 (2015) 359-386.

[2] W. Weiwei, Z. Zhuhuang, W. Shuicai, Z. Yanhua, Computational and Mathematical Methods in Medicine, 2016 (2016) 1-14.

[3] Y. Gao- Dai, Zhi-Cheng Li, Jia Gu, Lei Wang, Xing-Min Li, Published in: International Conference Image Processing (ICIP), 20th IEEE Explorer (2013) 1144-1147.
[4] M. Thomas, Deserno, Til Aach, Katrin Amunts, Walter Hillen, Torsten Kuhlen, Ingrid Scholl, Computer Science Research Development, 26 (2011) 1-3.

[5] Sonali Patil, Dr. V. R. Udupi, IOSR Journal of Electrical and Electronics Engineering (IOSRJEEE), 1, 4 (2012) 54-57.

[6] H. Isaac Bankman, "Handbook of medical image processing and analysis," Jadwiga Rogowska "Overview and fundamentals of medical image segmentation," Harvard Medical School, Elsevier Inc., p73. (2009).

[7] Faleh H. Mahmood, Nada A. Mahmood, Abdul Rahman H. Ismaeel, Iraqi Journal of Science, 58(3B), (2017) 1555-1564.

[8] M. Spiegel, D.Hahn, V. Daum, J.Wasza, J. Hornegger Compute Med Imaging Graph, 33, 1 (2009) 29-39.

[9] R. Muhammad Ghalib, Surbhi B., S. Jayapoorani, P. Udisha, International Journal of Engineering and Technology (IJET), 6, 1 (2014) 28-35.

[10] Mredhula. L M.A. Dorairangaswamy, Indian Journal of Computer Science and Engineering (IJCSE), 6, 2 (2015) 52-59.

[11] Faleh H. Mahmood, Wafaa A. Abbas, S. M. Ali, International Journal of Emerging Technology and Advanced Engineering Certified Journal, 4, 7 (2014) 509-517.

[12] HR. Shally and K. Chitharanjan, International Journal of Computer Science \& Engineering Technology (IJCSET), 4, 08 (2013) 1126-1132. 